\title{
Does the Compelled Cooperation Determine the Structure of a Complex Network? *
}

\author{
XUAN Qi(宣琦), LI Yan-Jun(李艳君)**, WU Tie-Jun(吴铁军) \\ Department of Control Science and Engineering, Zhejiang University, Hangzhou 310027
}

(Received 2 April 2007)

\begin{abstract}
Cooperation among individuals is considered to play an important role in the evolution of complex networked systems in physical, biological, economical and even epidemiological worlds, but its effects on the development of the systems is not so clear. We consider a specific kind of primal cooperation in a group of individuals, i.e., an individual never cooperates with others except when compelled to do so. The lowest level of compelled cooperation, in which cooperators share no message or resources, is investigated in the background of complex networks driven by the simple game rock-paper-scissors. Simulation results show that with the evolution of the systems, the cooperation will spread all over the networks, and finally results in systems with modular structures and a scale-free property.
\end{abstract}

PACS: 02.50.Le, 87.23. Kg, 89.65. Ef, 89.75. Hc

Over the past few years, complex network theory as a very useful tool to study the structure and behaviour of various complicated networked systems in physical, biological, economical and even epidemiological worlds has attracted much attention of researchers. ${ }^{[1-8]}$ Many complex networks in the real world have been modelled as graphs consisting of vertices and edges, and evolving from time to time in terms of pre-specified evolution rules. ${ }^{[1,4,7]}$ Investigation and analysis on the basis of these models have revealed some common properties of the systems, such as scale-free, ${ }^{[4]}$ small-world, ${ }^{[2]}$ hierarchical and modular structure, ${ }^{[6]}$ and so on.

All those models mentioned above are constructed based on an assumption: i.e., the nodes in a complex network act as independent entities, connecting each other in a homogeneous manner. Many facts in physics, biology, economy and other disciplines, however, do not match this assumption. For example, Colman ${ }^{[9]}$ pointed out that in biological systems, there are two types of essential relationships among individuals: competition and cooperation. Some individuals allied to compete with others for survival, driving the systems to evolve, and without cooperation, for instance, the mitochondria could not become one part of our body cells, and consequently there would not exist the humankind.

The origin and the evolution of cooperation in biological and social activities have been studied by many researchers. ${ }^{[10-27]}$ Two simple games, i.e. the Prisoner's Dilemma ${ }^{[10]}$ and the Snowdrift game, ${ }^{[11]}$ are frequently used by researchers in theoretical and experimental cooperation studies. In these games, individuals are considered to be selfish who maximize their own benefits at the expense of oth- ers. Recently, some other theories, such as kinselect, ${ }^{[20,21]}$ mutualism, ${ }^{[19,21]}$ altruism, ${ }^{[25,26]}$ indirect reciprocity, ${ }^{[24]}$ etc., are also introduced to explain the evolution of cooperation.

There are several researchers who have studied some kinds of cooperation in complex networks and obtained interesting results. ${ }^{[28-31]}$ For example, Anghel et al. ${ }^{[28]}$ used the minority game as a model for competition dynamics and showed that the influence network had a scale-free leadership structure; and Zimmermann et al. ${ }^{[30]}$ found that when the Prisoner's Dilemma was introduced into a complex network, the resultant network had a small-world property.

In this Letter, we consider a specific type of cooperation, compelled cooperation, in complex networks. Different from other kinds of cooperation, when we say an individual is compelled to cooperate with other individuals, it does not mean that the individual wants to cooperate with them for maximizing his own resources, mutualism or altruism; it just means that the individual must cooperate with others for his lack of resources used to compete with his opponents. In other words, the individual is forced to cooperate with others for survival. This type of cooperation is a kind of lower level activity compared with other cooperations in the common sense, so it may be closer to the origin of cooperation.

The inequality relationship between individuals in a compelled cooperation environment and the influence of this type of cooperation on the evolution of a system can be clearly explained by an evolutionary network modelled with a graph-theoretical approach. In this model, a network is made up of vertices and edges, where each vertex represents an individual and each edge the relationship between its

\footnotetext{
* Supported by the National Basic Research Programme of China under Grant No 2002CB312200.

**To whom correspondence should be addressed. Email: yjlee@iipc.zju.edu.cn

(C) 2008 Chinese Physical Society and IOP Publishing Ltd
} 
two end-vertices. Two kinds of relationship between two vertices are considered in the model: competition and cooperation. A pair of vertices that share a competitive edge is regarded as being in competition for resources. As the result of the competition, the loser releases an edge other than the edge between the loser and the winner randomly to the winner. If this released edge is also a competitive one, as is shown in Fig. 1(a), we can find that the loser loses the resources and a competitor who contended with the loser for the resources. If, on the other hand, the released edge is cooperative, then the loser loses the resources and a cooperator who shared the resources with the loser. In both the cases, the winner obtains the booty, i.e., the resources, and, additionally, a competitor or a cooperator. Otherwise, when the loser cannot pay the winner additional edges (in other words, the winner has all the neighbours that the loser owns), no matter which type of edges they are, the loser is compelled to cooperate with the winner in the remaining evolution process, as is shown in Figs. 1(b) and 1(c).

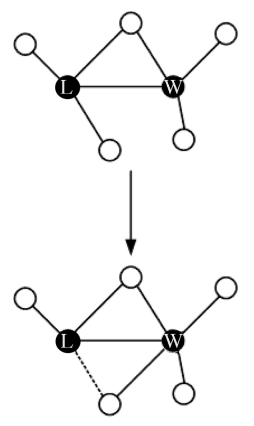

(a)

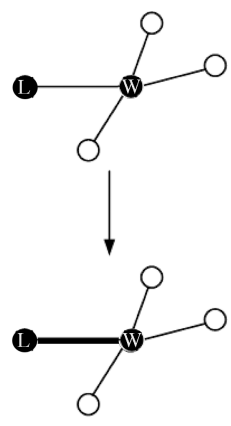

(b)

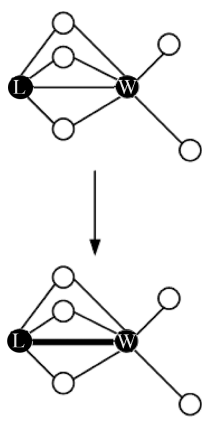

(c)
Fig. 1. Map of the compelled cooperation on network. The vertex marked $\mathrm{L}$ is the loser and the vertex marked $\mathrm{W}$ is the winner, two vertices with a common bold edge cooperate with each other and compete when with a common ordinary edge. (a) The loser gives an edge to the winner. (b) The loser has to cooperate with the winner for being alive. (c) The loser is compelled to cooperate with the winner because it cannot pay the winner of an additional edge.

The network will change its structure from time to time in this way, driven by a predetermined mechanism. Following the rules mentioned above, a competitive edge may be transferred to a cooperative one, and this change is irreversible. Logically, if all edges in the network have become cooperative, the network structure will not be able to change further. Equivalently we say the evolution of the network stops.

To describe how an individual cooperates with others, we divided the compelled cooperation into three evolutionary levels: not competing with each other (the lowest level), sharing message with each other (the middle level) ${ }^{[32]}$ and sharing resource with each other (the highest level). By the lowest level of coop- eration we just mean that cooperators do not compete with each other in system evolution processes, besides there is no any mutual assistance among them. In the middle level cooperation, on the other hand, the cooperators can share message to compete with their opponents, as is demonstrated in Fig. 2. In this figure vertex $\boldsymbol{a}$ has four competitors $(\mathbf{1}, \mathbf{2}, \mathbf{3}$, and $\mathbf{7})$ and two cooperators $(\boldsymbol{b}$ and $\boldsymbol{c}$ ). The cooperator $\boldsymbol{b}$, for example, helps $\boldsymbol{a}$ to ascertain its opponents $\mathbf{2}$ and $\mathbf{3}$, and the cooperator $\mathbf{c}$ helps $\boldsymbol{a}$ to ascertain $\mathbf{7}$, since they can share with $\boldsymbol{a}$ the knowledge about its competitors. Such message sharing can help the individual $\boldsymbol{a}$ make its competition strategy better, and it may be especially useful when its competition strategy is complex (when individuals are highly intelligent). When the cooperators can share resources in the competition with their opponents, we can find that they are in the highest level cooperation. In Fig. 3, there are five vertices (marked $\mathrm{C}$ and connected by bold edges) grouped to cooperate with each other. At a certain time, vertex $\boldsymbol{a}$ competes with vertex $\boldsymbol{c}$ and wins. Then the loser $c$ gives an edge $\overline{c d}$ (it can be regarded as a tribute) to a member of the group (not always the winner itself), here is $\boldsymbol{b}$. The simplest strategy in this type of compelled cooperation is to share resource with the members in a group with a uniform probability. This level of cooperation can help the cooperation group to maintain its integrality as the network evolves.

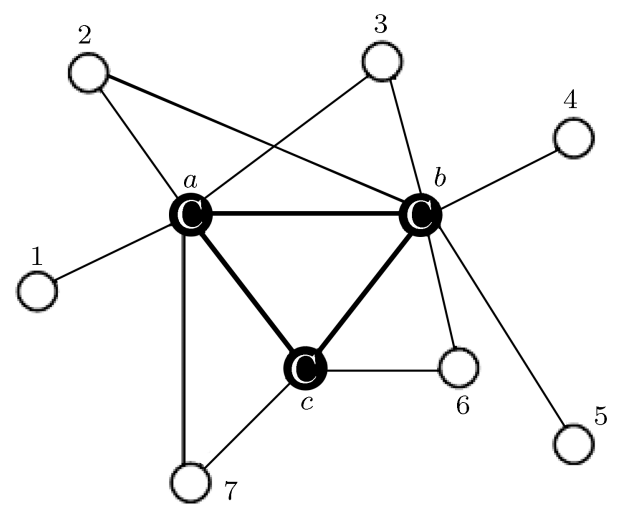

Fig. 2. The second level of cooperation: sharing signal with each other. The vertices marked $\mathrm{C}$ and connected by bold edges $(a, b, c)$ cooperate with each other.

There exist various kinds of mechanisms to drive a network to evolve, the simplest one may be the random game, rock-paper-scissors. By this game we select a winner from a pair of competitive vertices randomly. It is interesting to study the influence of the different levels of compelled cooperation on the evolution of a network system driven by the simplest game. However, for the limitation of this Letter, we simply investigate the effect of the lowest level of cooperation, not competing with each other. A network is driven to evolve in the following three steps: 
Step 1. Initialization. The evolution begins at an initial random network with $N$ vertices and $M$ competitive edges.

Step 2. Compete or cooperate. At each time, two opponents (the vertices with a common competitive edge) are selected randomly to play the game rockpaper-scissors. The loser gives an edge to the winner (the loser loses a neighbour and the winner gets it), or cooperates with the winner.

Step 3. Repetition. Step 2 is repeated until each two connected vertices in the network cooperate with each other.

Three different networks have been tested in terms of the evolution rules mentioned above. The initial sizes of the networks are set as follows: the first network has 200 vertices and 419 edges; the second 200 vertices and 980 edges; and the last 200 vertices and 3928 edges. When all the vertices in those networks are connected as cooperators (this means that there is not any competition in the networks), the evolution processes stop and we investigate the structures of the resultant networks.

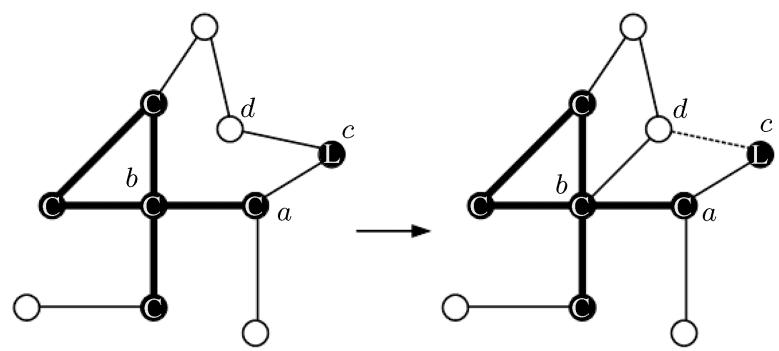

Fig. 3. The highest level of cooperation: sharing resource with each other. The vertices marked $\mathrm{C}$ and connected by bold edges cooperate with each other. Vertex $a$ competes with vertex $c$ and wins, and the loser $c$ gives an edge $\overline{c d}$ to $a$ 's cooperator $b$.
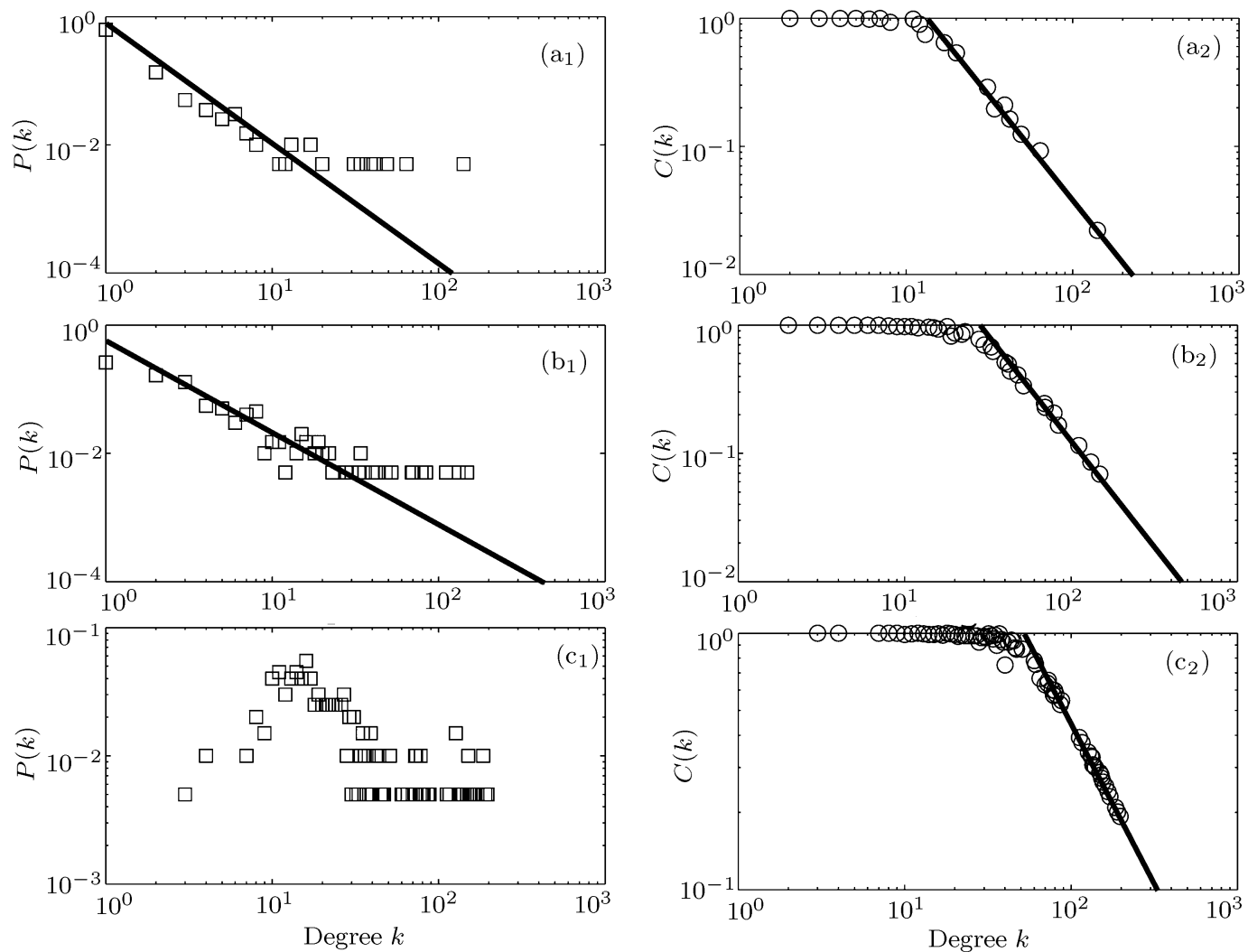

Fig. 4. Plots of the resulting network's degree distribution $P(k)$ (left) and the clustering function $C(k)$ (right): (a) from a random network with 200 vertices and 419 edges, (b) from a random network with 200 vertices and 980 edges, (c) from a random network with 200 vertices and 3928 edges.

We find that the first and the second resultant networks, as shown in Fig. 4(a $\left.\mathrm{a}_{1}\right)$ and $4\left(\mathrm{~b}_{1}\right)$, have scale-free properties; and in the third network, the edges are distributed nearly uniformly among the vertices (refer to Fig. $\left.4\left(c_{1}\right)\right)$. These results are reasonable and explainable. We take a human society as an example. Suppose that in the society (network), there is initially a lack of resources (edges). That is the first case in our simulation. Then the individuals who initially possess more resources than others will have more opportunities to compete with them. Thus with the evolution of the society, most of the resources will be gradually concentrated to a few of individuals (a few vertices possess most of the edges in the network), and other 
individuals get poorer and poorer (other vertices lose their edges). When the initial resources in the society increase (this is the second case in our simulation), the gap between the poor and the rich will be reduced as the society evolves (the slope of the line in Fig. $4\left(b_{1}\right)$ is smaller than that in Fig. $\left.4\left(\mathrm{a}_{1}\right)\right)$. Furthermore, when the resources in the society are abundant enough (the third case in our simulation), all the individuals in the network will have nearly equivalent chances to compete for resources. Thus the evolution of the society will result in a strong middle class, i.e., most of the resources will distribute uniformly over most of the individuals in the society (most of the vertices possess most of the edges uniformly, as is shown in Fig. $\left.4\left(c_{1}\right)\right)$. We also find that all three resultant networks have modular structures shown in Figs. 4( $\left(\mathrm{a}_{2}\right)$, $\left(b_{2}\right)$, and $\left(c_{2}\right)$, which are very similar to many networks in the real world.

In conclusion, the method we propose here is designed to model complex networks in the presence of compelled cooperation activities and to investigate the influence of the cooperation on the evolution of the systems. This model provides a basis for the behaviour analysis and prediction of a wide range of realworld networked systems, such as biological, social, and economical systems, in which cooperation plays a very important rule. Many interesting phenomena in the world, e.g., the emergence of multicellular lives and their scale-free structures, can be explained or predicted by the proposed model. In an environment lack of resources, for example, unicellular lives with less resources were forced to congregate with those possessing more resources. In a long period of evolution such kind of 'cooperation' leads to the naissance of multicellular lives. ${ }^{[16,17]}$ To a certain extent we can say that just the resource shortage is the motivity of evolution, under the assumption that there is only the lowest level of cooperation.

We must point out that those conclusions are drawn from the simulation results on the basis of the specific kind of cooperation by which it only means that the individuals do not compete with each other, besides there is not any substantive collaboration among them. When the cooperators are able to share message or resources in their collaboration, however the consequence may be changed. Investi- gating the results adopting higher cooperation rules and different system evolution mechanisms is a very interesting topic. Our future research will follow this guideline.

We would like to thank all the members in our research group in the Institute of Intelligent Systems and Decision Making, Zhejiang University at Yuquan Campus, for the valuable discussions about the ideas presented here.

\section{References}

[1] Albert R and Barabási A -L 2002 Rev. Mod. Phys. 7447

[2] Watts D J and Strogatz S H 1998 Nature 393440

[3] Barrat A and Weigt M 2000 Eur. Phys. J. B 13547

[4] Barabási A-L and Albert R 1999 Science 286509

[5] Barabási A-L and Oltvai Z N 2004 Nature 5101

[6] Ravasz E, Somera A L, Mongru D A, Oltvai Z N and Barabási A-L 2002 Science 2971551

[7] Xuan Q, Li Y J and Wu T J 2006 Phys. Rev. E 73036105

[8] Strogatz S H 2001 Nature 410268

[9] Colman A M 1982 ooperation and Competition in Humans and Animals (Wokingham: Van Nostrand Reinhold)

[10] Nowak M A, Sasaki A, Taylor C and Fudenberg D 2004 Nature $\mathbf{4 2 8} 646$

[11] Hauert C and Doebeli M 2004 Nature 428643

[12] Roberts G and Sherratt T N 1998 Nature 394175

[13] Taylor P D and Day T 2004 Nature 428611

[14] McNamara J M, Barta Z and Houston A I 2004 Nature 428 745

[15] Ackermann M and Chao L 2004 Curr. Biol. 14 R73

[16] Rainey P B and Rainey K 2003 Nature 42572

[17] Vellcer G J and Yu Y -T N 2003 Nature 42575

[18] M-Gibbons M and Adams E S 2002 Science 2982146

[19] Palameta B and Brown W M 1999 Animal Behavior 57 F1

[20] West S A, Pen I and Griffin A S 2002 Science 29672

[21] C-Brock T 2002 Science 29669

[22] Brandt H, Hauert C and Sigmund K 2006 Proceedings of the National Academy of Sciences 103495

[23] Roughgarden J, Oishi M and Akcay E 2006 Science 311 965

[24] Nowak M A and Sigmund K 2005 Nature 4371291

[25] Fehr E and Fischbacher U 2003 Nature 425785

[26] Fowler J H 2005 PNAS 1027047

[27] Nowak M A and Sigmund K 2002 Nature 418138

[28] Anghel M, Toroczkai Z, Bassler K E and Korniss G 2004 Phys. Rev. Lett. 92058701

[29] Santos F C and Pacheco J M 2005 Phys. Rev. Lett. 95 098104

[30] Zimmermann M G, Eguíluz V M and Miguel M S 2004 Phys. Rev. E $69065102(\mathrm{R})$

[31] Holme P, Trusina A, Kim B J and Minnhagen P 2003 Phys. Rev. E 68 030901(R)

[32] Gintis H, Smith E A and Bowles S 2001 J. Theor. Biol. 213103 